\title{
Hippo(crates): An integrated atlas for natural product exploration through a state-of-the art pipeline in chemoinformatics
}

\author{
LOUIS PAPAGEORGIOU $^{1}$, ATHENA ANDREOU ${ }^{1}$, ELIAS CHRISTOFORIDES ${ }^{2}$, \\ KOSTAS BETHANIS ${ }^{2}$, DIMITRIOS VLACHAKIS ${ }^{1}$, TRIAS THIREOU ${ }^{1}$ and ELIAS ELIOPOULOS ${ }^{1}$ \\ ${ }^{1}$ Department of Biotechnology, Genetics Laboratory, School of Applied Biology and Biotechnology, \\ Agricultural University of Athens; ${ }^{2}$ Department of Biotechnology, Physics Laboratory, \\ School of Applied Biology and Biotechnology, Agricultural University of Athens, 11855 Athens, Greece
}

Received August 29, 2021; Accepted November 4, 2021

DOI: 10.3892/wasj.2021.136

\begin{abstract}
Modern drug discovery and pharmaceutics benefit from nature. Natural products (NPs) are used as a source of therapeutic agents with beneficial uses. Currently, there is considerable interest in the exploration of NPs for drug discovery and continuous investigations on the therapeutic claims and mechanisms of herbal medicines. To date, approximately one million NPs have been isolated and subjected to experimental assays to evaluate quantitative biological activities. This renders the use of an integrated database to assemble and correlate this valuable information from the literature, experimental studies and databases necessary. Although databases contain a large volume of information, it is frequently difficult and complex, even in well-organized databases, to extract the required information. Novel databases must be accompanied by efficient algorithms and techniques in order to extract beneficial knowledge by a simple query. The Hippo(crates) database aims to fill this gap in the field of chemoinformatics and natural products by providing retrieval not only linked to the Hippo(crates) database, but also to other worldwide chemical and biological databases. Part of the OPENSCREEN-GR project, the Hippo(crates) Database Graphical User Interface (HDGUI) web server was developed to provide a user-friendly access interface, integrating annotated information of NP origin (sources and species), biological activities, physicochemical properties, linear and 3D chemical structure, as well as relative terms that correlate chemical
\end{abstract}

Correspondence to: Professor Elias Eliopoulos, Department of Biotechnology, Genetics Laboratory, School of Applied Biology and Biotechnology, Agricultural University of Athens, 75 Iera Odos, 11855 Athens, Greece

E-mail: eliop@aua.gr

Key words: natural products, chemoinformatics, data mining, semantic, pharmacophores, database compounds and their use. In its current version (V1.0), the Hippo database provides 45,300 NPs, NP derivatives and synthetic compounds, which are separated into 32 major categories, including biological or medicinal properties. In the database, 22,830 NP source organisms are correlated, with $>100,000$ terms, including biological pathways, target organisms, target diseases, target types, target proteins and pathogens, and 6,070 three-dimensional structures of NP target proteins. For each entry, a cluster with similar compounds and a ligand-based or structure-based pharmacophore model is provided. The portal is designed as an easy-to-use web tool where the user can easily search, extract and correlate information and data for natural product chemical compounds through various fields, such as categories, keywords, targets, species, or two-dimensional or three-dimensional similarity structure in the Hippo(crates) atlas of the NP database.

\section{Introduction}

History: Overview of natural products (NPS). NPs are primary and secondary metabolites produced and used by living organisms for defending mechanisms or adapting actions. These molecules have been naturally selected and modified for millions of years to acquire specificity and cover a wide range of biological mechanisms, depending on the originating species, the environment, and the specific biological action involved in the corresponding organism (1). Considering these beneficial features, NPs have been used as therapeutic agents for thousands of years from the beginning of rational medicine and to date, continue to be the most important source of novel candidate therapeutic agents in the pharmaceutical industry $(2,3)$.

NPs, derived mostly from herb plants, have been used as the major source of therapeutics for traditional medicine throughout history and continue to be the basis for a number of pharmaceuticals currently used (4). The pharmaceutical properties of herb plants were described on Assyrian clay tablets dating back to 2000 B.C. and were also reported in ancient Greek culture by Hippocrates (400 B.C.) and 
Galinos (160 A.C). Currently, NPs are the principal source of compounds for modern medicine, and the extent of the mining of such compounds has increased during the 20th century (5). The advantage of the use of NPs for the development of novel drugs and other chemicals derives from their innate affinity for biological receptors and their interaction in a number of biological functions. NPs have provided the most effective antiparasitic, anti-infection and antidiabetic compounds known to date (5-7).

Over the past decades, huge libraries of fractionated NPs have been screened with impressive hit rates in several diseases and pathogenic conditions. Of note, a number of cases are known where the crude biological extract is more pharmacologically effective against the purified most active chemical compound from this extract (8). In several instances, other compounds present in the extract with no pharmacological activity function synergistically with the primary compound (9). To date, the effective compounds are screened using advanced cell-based assay techniques, the candidate targets in the cell are probed, and possible synergies are identified $(10,11)$.

Use of NPs in biology, pharmacology and medicine. Traditional medicines and NPs provide valuable insight towards the discovery of novel medicinal agents. Crude biological extracts may help to enlarge the drug discovery paradigm from 'identifying novel entity drugs' to 'combining existing agents' and may even direct the combinations between such NP-derived agents $(12,13)$. Recent structural comparisons between NPs and modern drugs or candidates identified $35 \%$ of NPs that are structurally similar or identical in structure with modern therapeutic agents (14). Although modern pharmaceutical drugs were born from botanical medicine and are mainly NPs, synthetic approaches to drug development have more recently turn out to be standard. Based on recent studies on human drugs introduced between the early 1980 s until $2014,62 \%$ of the new small-molecule drugs were either NPs, derived from NPs (often semi-synthetically), and NPs derived from pharmacophores (considered as NPs analogs) $(4,15)$. However, the synthetic combinatorial chemistry and high-throughput screening (HTS) of potential modern drug targets disconnected the link between NPs and medicines. In the early 1990s, the pharmaceutical industry turned towards the HTS of chemical libraries against potential pharmacological targets, while the screening of NPs was diminished. There was a feeling that NPs were an 'obsolete science' and not a scientifically valid paradigm for the modern discovery of novel drugs or chemicals. More recently, modern pharmaceutical research has acknowledged this oversight and stimulated new interest in the potential of NPs as novel pharmaceutical agents (15). In a great effort to recover and recombine the abandoned fragmented information of the pharmaceutical properties of NPs, the Hippo(crates) NP database, currently containing multidimensional knowledge based on the collected and analyzed and cross-correlated information for each NPs, was developed.

Use of chemoinformatics and new goals. Chemoinformatics provide computer methods for the organization, analysis and visualization of chemical information, and is used extensively in drug discovery and development. It is a rapidly evolving field, particularly due to the advent of high-throughput experimental techniques, the widespread availability of public databases, and the development of machine learning algorithms $(16,17)$. Successful results of chemoinformatics approaches, such as Quantitative Structure-Activity Relationship (QSAR) or Quantitative Structure-Property Relationship (QSPR) and drug design, depend critically on the quality of data and the representation of chemical structure information through chemical descriptors and high-dimensional vectors termed fingerprints. Several fingerprint methods and similarity coefficients are used in similarity-based virtual screening applications in order to identify database compounds with probably similar bioactivity to a query compound (18).

Pharmacophore is another concept integral to computer-aided drug design. It is the ensemble of steric and electronic features necessary to ensure the optimal supramolecular interactions with a specific biological target structure and to trigger (or to block) its biological response (19). Pharmacophore models can be derived from experimentally determined protein-ligand complexes (receptor-based pharmacophores), or from known active compounds (ligand-based pharmacophores) $(20,21)$.

Recently, polypharmacology, the ability of a single agent to interact with multiple receptors and modulate several processes, has drawn attention (22). Apart from studying drug side-effects, polypharmacology facilitates the repurposing of 'old' drugs to treat both common and rare diseases, hopefully reducing costs and accelerating drug development $(23,24)$. Ligand-based computational methods of predicting small molecule's unknown targets involve similarity searches in databases containing information on the activity of compounds and their protein targets (25).

Hippo(crates), an updated atlas of NPs. Different databases have been shared in recent years, providing information required to develop the exploration and exploitation in NPs. As expected, each database has been specialized in a different field and presents the NPs from a different point of view, including DrugBank, Natural Product Activity and Species Source (NPASS), NPCARE and Open National Cancer Institute (NCI) (Table I) $(26-30,32)$. The Hippo(crates) database aims to facilitate the combination and correlation of different fields of NP knowledge in a unified platform, providing knowledge of each NP in an updated atlas of NPs. In the same direction, several tools and algorithms have been incorporated in the Hippo(crates) Database Graphical User Interface (HDGUI) in order to export collective results and provide beneficial knowledge of individual NPs or a group or category.

The Hippo(crates) database aims to assist the pharmaceutical research for novel potential candidate pharmacological agents and pharmacological targets. The user can perform searches using the HDGUI with a combination of several preset parameters, features, properties and keywords related to the NPs and chemical compounds. The HDGUI applies various filtering, processing and annotation techniques towards identifying and visualizing the most probable dominant NPs and chemicals based on the user preset parameters. The HDGUI identifies all the candidate NPs using the up-to-date curated Hippo(crates) database and provides each chemical compound information guided by explanatory information from the 
Table I. The six source databases and studies that were used for the synthesis of the Hippo(crates) database.

\begin{tabular}{|c|c|c|c|c|}
\hline $\mathrm{A} / \mathrm{A}$ & Source/(Refs.) & Sample & Common identifiers & Dataset \\
\hline 1 & Selleckchem & 16550 & $\begin{array}{l}\text { - CAS Number } \\
\text { - Canonical SMILE }\end{array}$ & $\begin{array}{l}\text { Natural products } \\
\text { Synthetic drugs }\end{array}$ \\
\hline 2 & Open NCI & 15000 & $\begin{array}{l}\text { - CAS Number } \\
\text { - InChIKey }\end{array}$ & $\begin{array}{l}\text { Natural products } \\
\text { Synthetic drugs }\end{array}$ \\
\hline 3 & DrugBank & 700 & $\begin{array}{l}\text { - Canonical SMILE } \\
\text { - InChIKey }\end{array}$ & Natural products \\
\hline 4 & NPCARE & 9100 & - Canonical SMILE & Natural products \\
\hline 5 & NPASS & 30000 & $\begin{array}{l}\text { - Canonical SMILE } \\
\text { - CID }\end{array}$ & Natural products \\
\hline 6 & Newman and Cragg (15) & 1376 & - Name & $\begin{array}{l}\text { Natural products } \\
\text { Synthetic drugs }\end{array}$ \\
\hline
\end{tabular}

Open NCI, Open National Cancer Institute; NPASS, Natural Product Activity and Species Source.

annotation and data mining analyses, as well as direct links to several online databases, such as PubChem (https://pubchem. ncbi.nlm.nih.gov/), Protein Data Bank (https://www.rcsb.org/) and a chemical 3D viewer.( https://molview.org/).

\section{Data: From collection to clustering}

Data collection and filtering. The NP derivatives and synthetic compounds with available experimentally-determined quantitative activity, chemical, physicochemical properties and relative information were extracted from the Selleckchem available catalog (https://www.selleckchem.com/) (31), the Open NCI database (https://cactus.nci.nih.gov/download/nci/) (26,32), the DrugBank database (https://go.drugbank.com/) $(27,28,33)$, the NPCARE database (http://silver.sejong.ac.kr/npcancer/) (30) and the NPASS database (http://bidd.group/NPASS/) (29) by using combinations of keywords related to the term 'natural products'. Moreover, the final dataset with the results of the NP chemical study by Newman and Cragg (15) has been included in the present study. A detailed content comparison among the six extracted chemical datasets is provided in Table I. All the retrieved information has been analyzed towards identifying common chemical characteristic identifiers in the extracted chemical datasets, including 'Name', 'Canonical SMILES', 'CAS registry number', 'International Chemical Identifier (InChIKey)' and PubChem id 'CID' $(34,35)$. The Hippo(crates) database has been synthetized from the described chemical datasets, and each chemical entry has been checked to be unique in the demo version of the database by using the common chemical characteristic identifiers information (Fig. 1). All additional information from the common chemical entries between the six extracted chemical datasets has been included in the unique entries of the Hippo(crates) database for each duplicate chemical as described in the data annotation and processing step. The main pipeline of the described procedure is presented in Figs. 1 and 2.

Data annotation and processing. Hippo(crates) database entries have been annotated with information from several fields contained in the PubChem Database by using one or a combi- nation of the four primary identifiers (Name, CAS Number, InChIKey and CID) describing each chemical compound (Fig. 1). More specifically, from the PubChem database, the extracted information contains the name, synonym_name, canonical_SMILES, InChiKey, Formula, Molecular_Weight, alogp and Xlog (Fig. 2). The second level of annotation has been performed with the use of the four common identifiers on the primary datasets of the chemical compounds. According to the type of knowledge contained in the primary datasets, information has been extracted and annotated for each entry, including target_protein, target_disease biological_pathway, target_organism and source_organism (Fig. 2). Subsequently, data mining and semantic analyses have been performed using the Matlab Bioinformatics toolbox towards identifying the major representative 'ontologies' per specific field with the entries being characterized on the basis of the recognized ontologies in categories (Fig. 2 and Table II) $(36,37)$. Through the data annotation, the major goal was to find the hidden correlations between the chemical compounds and their connection with other international chemical and structural databases. Chemical compounds have been linked to the PubMed database using the 'CID' and with the Protein Data Bank using the 'InChIKey', respectively (Fig. 2). Last but not least, specific chemical properties have been calculated using the program MOE, including 'hba', 'hbd', polarsurface, 'rings' and 'heavyatoms' (Fig. 2) $(38,39)$.

Data clustering and pharmacophore design. Compound fingerprints were calculated using the CACTVS Chemoinformatics Toolkit (40). The Sphere Exclusion algorithm $(41,42)$ complemented with Tanimoto coefficient (43) was applied to select diverse subsets from the Hippo(crates) database. This procedure was repeated several times using different parameter sets in order to identify the optimal thresholds and separate the dataset in internal clusters. This was followed by pharmacophore determination for the NPs and chemical compounds that were annotated into the Hippo(crates) database using the analyzed information from the previous processing step. In the present study, both ligand-based and structure-based pharmacophore model design was performed 


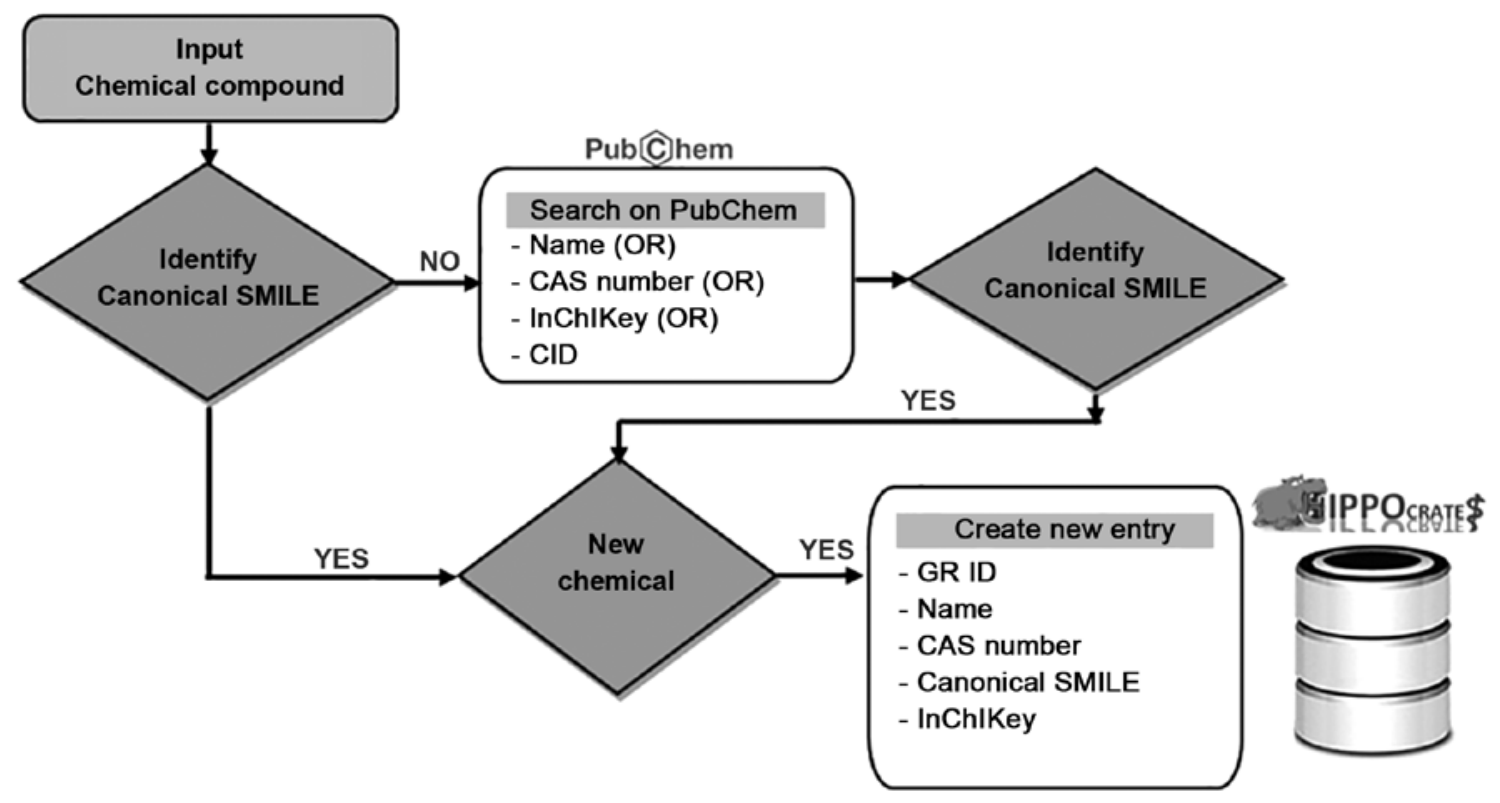

Figure 1. Data collection and filtering pipeline of the Hippo(crates) database.

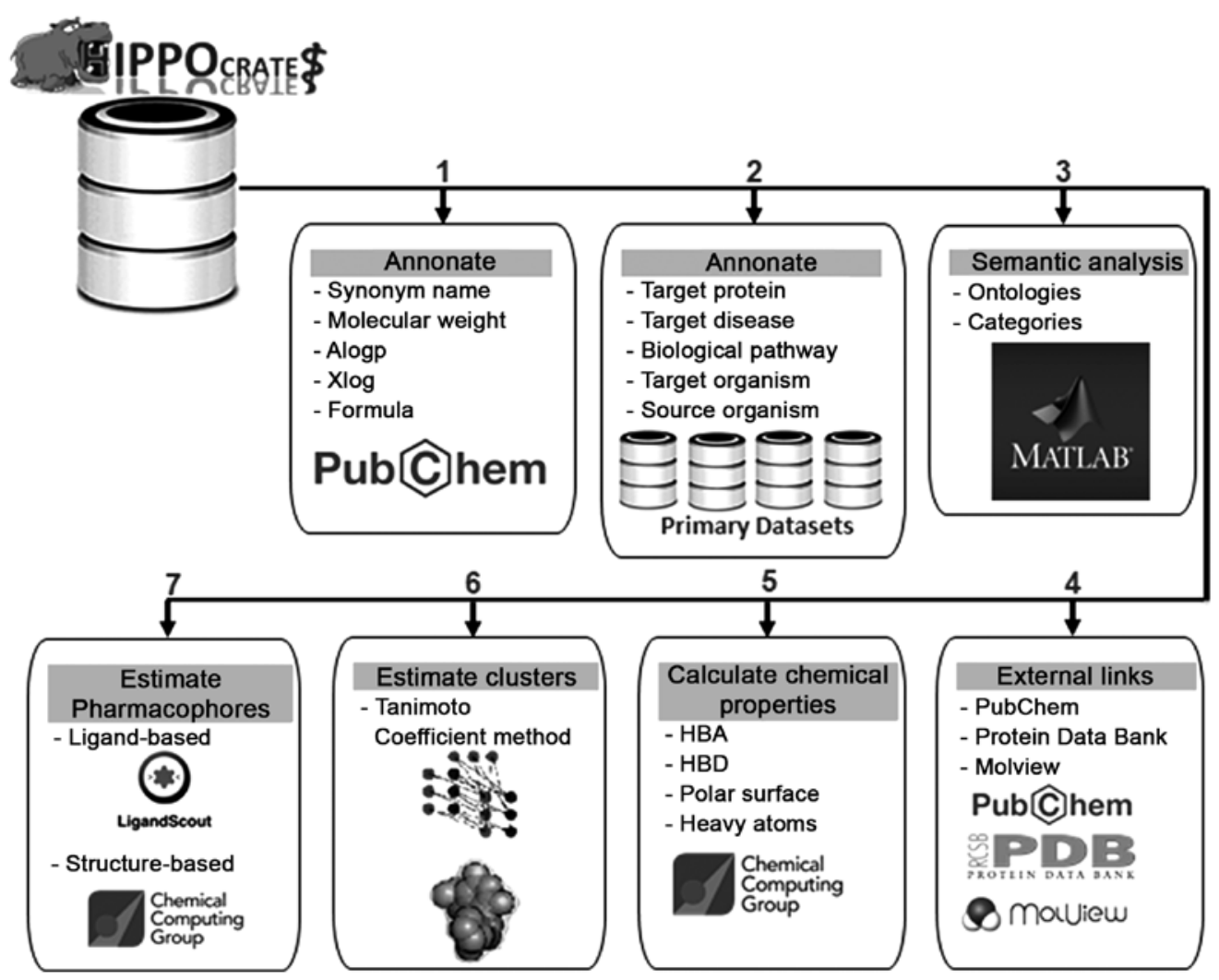

Figure 2. Data annotation and processing pipeline of the Hippo(crates) database.

(Fig. 2). The Tanimoto coefficient for clustered compounds was employed to generate ligand-based pharmacophores using LigandScout (44). The structure-based pharmacophores were constructed using the chemical compounds that were co-crystalized with proteins in experimentally determined complexes from the Protein Data Bank (PDB). A specialized analysis has been setup towards estimating the corresponding protein cavities for each chemical compound and then using their characteristic features for designing the corresponding pharmacophore models using MOE $(45,46)$.

\section{Structure of the Hippo(crates) database}

HDGUI) webserver. The Hippo(crates) database of NPs and chemical compounds is publicly available online at http://www. openscreen.aua.gr/login.php. The HDGUI runs on a Secure 
Table II: List of the 32 major categories present in the Hippo(crates) database.

\begin{tabular}{rlll}
\hline A/A & \multicolumn{1}{c}{ Category } & A/A & Category \\
\hline 1 & Natural Product & 17 & Epigenetics \\
2 & Anticancer & 18 & FDA Approved \\
3 & Antidiabetic & 19 & GPCR related \\
4 & Antiinfection & 20 & Immunology inflammation \\
5 & Antibacterial & 21 & Inhibitors \\
6 & Antihypertensive & 22 & Ion channels related \\
7 & Antiviral & 23 & Kinase_inhibitor \\
8 & Antiparasitic & 24 & MAPK inhibitor \\
9 & Antifungal & 25 & Metabolism compound \\
10 & Antiulcer & 26 & Neuronal signaling \\
11 & Apoptosis & 27 & PI3K \\
12 & Autophagy & 28 & Protease inhibitor \\
13 & Bioactive compound & 29 & Pdb_related \\
14 & Clinical & 30 & Stem cell signaling \\
15 & Calcium metabolism & 31 & Target selective \\
16 & Drug repurposing & 32 & Tyrosine kinase inhibitor \\
\hline
\end{tabular}

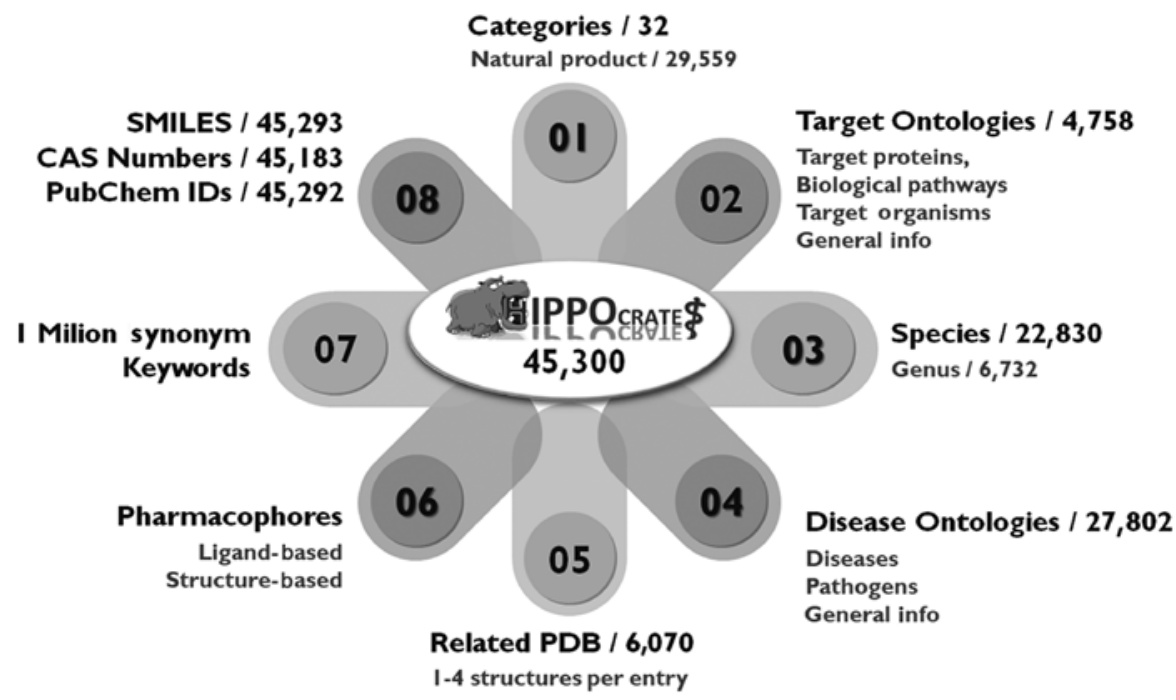

Figure 3. Hippo(crates) database contents.

HTTP Apache web server hosted at the HDGUI web server, using the LINUX Operating System, Apache Technology, PHP, JavaScript, R, and parallel computing architecture on the computing facility of the School of Applied Biology and Biotechnology at the Agricultural University of Athens (AUA). HDGUI has been designed in a way to enable the user to retrieve NPs and chemical compounds through various developed toolboxes. Additionally, specialized toolbars that have been added to the interface enable the user to make 2D and 3D chemical similarity searches (molecular similarity score of $\geq 0.9$ ) in the PubChem database using the extracted chemical SMILES from the Hippo(crates) database $(47,48)$.

The Hippo(crates) database. The Hippo(crates) database is an integrated resource for NPs, chemical compounds derived from NPs and chemical compounds considered as NPs analogs, and other chemical compounds. The Hippo(crates) database currently holds 45,300 entries, which are divided into 32 major categories, as presented in Table II. Moreover, from the annotation and semantic analyses, 45,293 chemical SMILES, 45,183 CAS numbers, 45,292 PubChem IDs, 4,758 target ontologies, 27,802 disease ontologies, 22,830 source organisms and 6,070 connected protein 3D structures from the PDB have been correlated (Fig. 3). The information within the database is structured in 32 different fields, and the knowledge is organized in a specific manner in order to serve the webserver application immediately and in a timely manner.

\section{Functionality of the Hippo(crates) database}

Chemical clusters and pharmacophores. The Hippo(crates) database provides a well-organized atlas of interconnected 


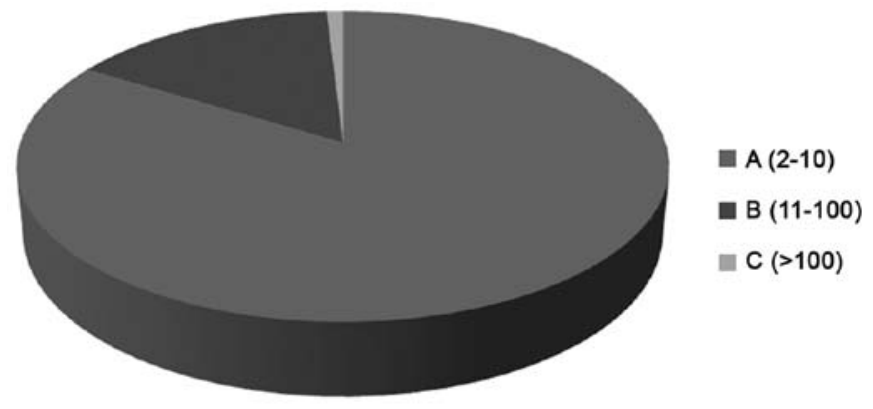

Figure 4. Tanimoto coefficient ' 80 ' chemical clusters. (A) Chemical clusters with maximum 10 chemical compounds per cluster. (B) Chemical clusters with minimum 11 and maximum 100 members. (C) Chemical clusters with at least 111 members.

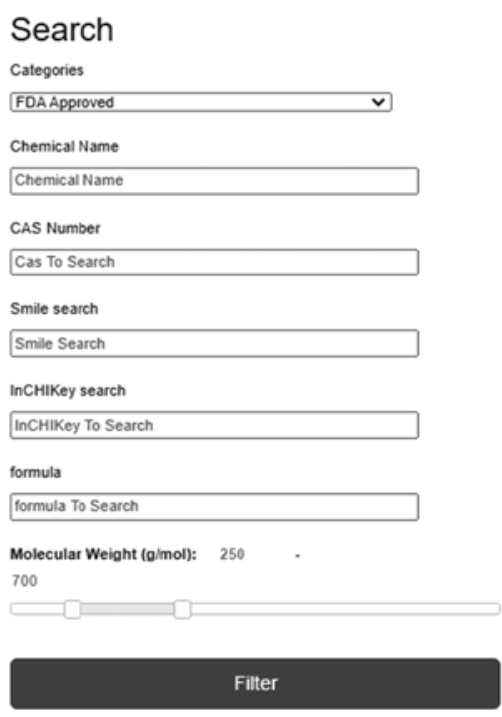

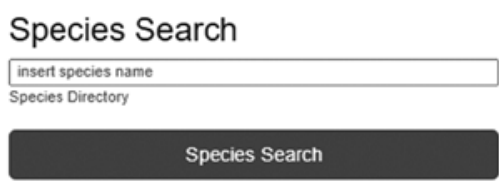

\section{Target Search}
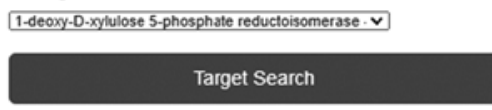

Disease or Target Organism

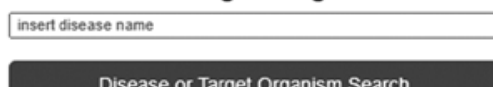

Disease or Target Organism Search

\section{Smart Search}

Insert separated keywords (maximun four) and click "Search"

insert keywords

\section{Search}

\section{Blast Chem}

\section{insert a canonical SMILE}

Blast chemical canonical smile in Pubchem

2D Similarity Search

$3 \mathrm{D}$ Similarity Search

\section{Pharmacophore Search}

Figure 5. Hippo(crates) database graphical user interface (HDGUI) webtools.

NPs and other chemical compounds using both advanced bioinformatics and chemoinformatics techniques. The contents of the database were analyzed using specialized techniques such as the Tanimoto coefficient for the analysis of chemical compounds (43). Based on the results, 3,046 different clusters were identified containing 2 to 1,600 chemical compounds (Fig. 4). A second level of chemical analysis was performed towards generating the representative pharmacophore models for each entry (Fig. 2). Specifically, 2,100 ligand-based and 673 structure-based pharmacophore models were constructed. The results of the chemoinformatic analysis are interlinked in the Hippo(crates) database and are accessible through a customized toolbox.

\section{Usability and applications}

HDGUI webserver. The HDGUI webserver aids the chemical and medical experts, pharmacists and other users in searching and identifying NPs, NP-derived and other synthetic chemicals with identified chemical properties through a characteristic set of keywords and ontologies. This is achieved through filtering web tools and the summarized knowledge under 'key' terms is presented in smart lists. Users are able to perform complex filtering operations using chemical properties, fingerprints, disease, target proteins, biological pathways, source organisms and several other specific keywords under specific domain ontologies. In addition, the HDGUI webserver enables users who may not be familiar with chemical molecular structures (Fig. 5) to be able to discover, filter and classify, and easily present NPs and other chemical compounds contained in the Hippo(crates) database. Furthermore, users are able to select and list efficiently chemical compounds that are associated with 'key' terms, domain ontologies, or characteristic fingerprints (SMILES).

The HDGUI filtering options are separated into seven major webtools (Fig. 5). The default simple search provides the ability to the user to discover chemical compounds based on a specific international identifier such as chemical name, SMILES, CAS number and InCHIkey or characteristic chemical properties, or a combination of those terms. The second web tool named 'Species Search' provides the user with the ability to extract chemical compounds that are extracted from a specific source organism such as plants, herbs, fungi, flatworms and other living organisms. The species search webtool is well-organized with a directory and smart list in an effective manner from which the user can identify the main genera and the species contained in the Hippo(crates) database. The third and the fourth web tools named 'Target Search' and 'Disease or Target Organism' provide the user with lists of key terms identified, classified and summarized from a semantic analysis 


\section{A}

\section{Pharmacophore Search}

$C 1=C C 2=C(C=C(C=C 2 B r) B r)$ ) $N=C 1$

\section{Pharmacophore Search}

\section{B}

\section{Back to menu}

Pharmacophore Search

\begin{tabular}{|c|c|c|c|c|c|c|c|}
\hline GRID & Smile & Name & PubChem & PDB & $\begin{array}{l}\text { Ligand- } \\
\text { based }\end{array}$ & Structural-based & Tanimoto80> \\
\hline GR1317 & $\mathrm{C} 1=\mathrm{CC} 2=\mathrm{C}(\mathrm{C}(=\mathrm{C}(\mathrm{C}=\mathrm{C} 2 \mathrm{Br}) \mathrm{Br}) \mathrm{O}) \mathrm{N}=\mathrm{C} 1$ & Broxyquinoline & Pubchem & $\begin{array}{l}6 \mathrm{FUK} \\
2 X X Z \\
3 \mathrm{~N} J Y \\
3004\end{array}$ & Download & 3od4_1_phar.ph4 & Download \\
\hline
\end{tabular}

Figure 6. An example of the 'Pharmacophore Search' web tool. (A) The input query of a specific SMILES in the web tool. (B) The output results based on the input query SMILES.

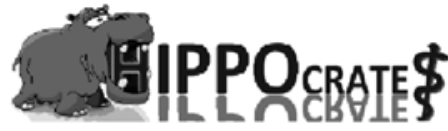

\section{Filter Details}

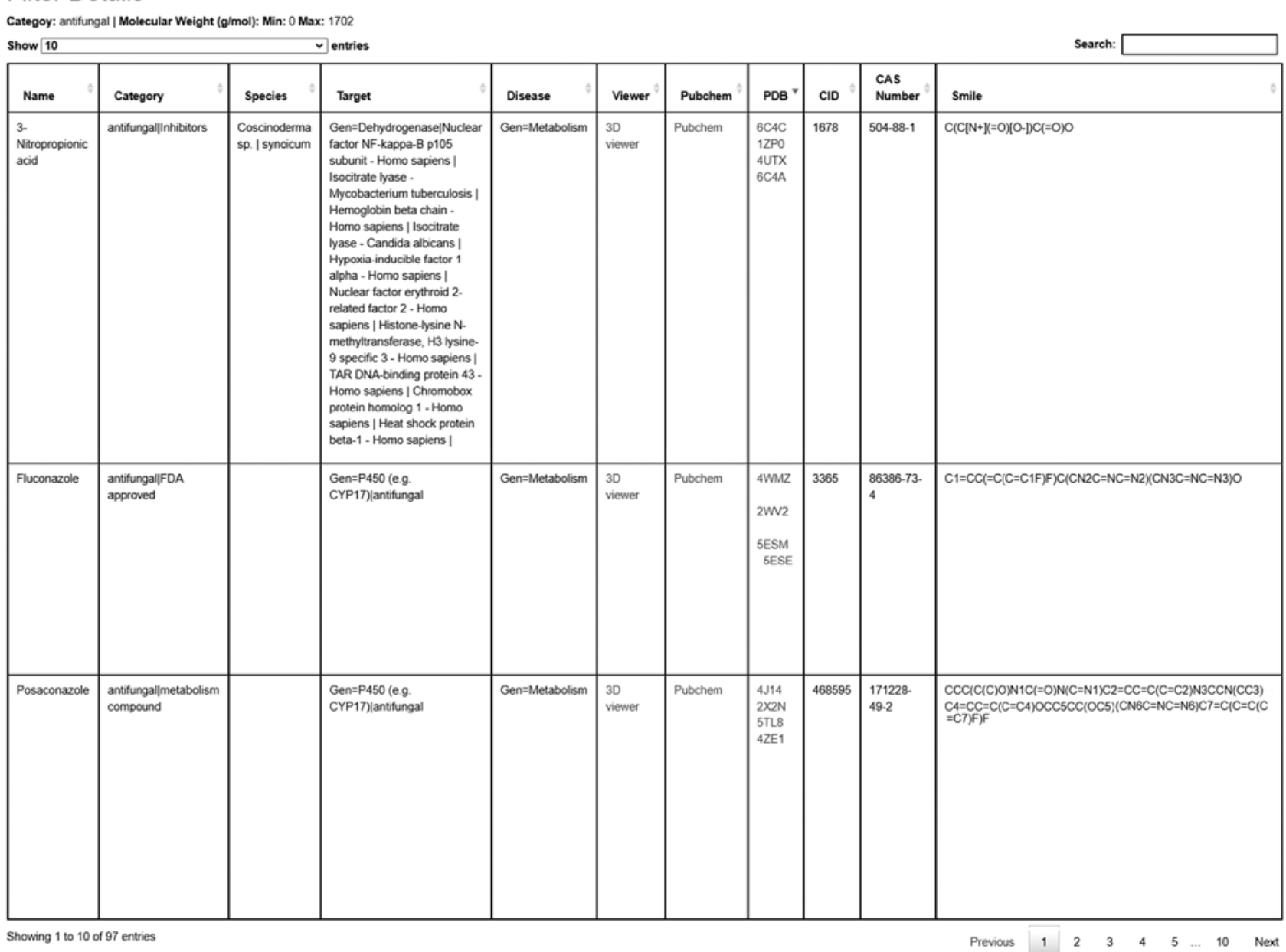

\section{Back to menu}

Figure 7. An example of the Hippo(crates) database graphical user interface (HDGUI) based on a simple filtering search. 
study. By clicking a specific term from the lists, the user is able to discover a cluster of chemical compounds that are correlated with this specific term. The fifth webtool name 'Smart Search' enables the user to perform a filtering search in the contents of the Hippo(crates) database based on a number of specific keywords of preference. The sixth webtool named 'Blast Chem' is an integrated service from where the user selects similar 2D and 3D chemical compounds with a 95\% similarity cutoff from the PubChem database using the SMILES identifier. Finally, the last webtool named 'Pharmacophore Search' provides the user with the option to discover the possible ligand-based and structure-based pharmacophore models by using a SMILES entry of a chemical compound. Furthermore, in this webtool, the user can download the dataset of the cluster of the chemical compounds from which the pharmacophoric model has been generated (Fig. 6).

The HDGUI output is an HTML file that describes the chemical compounds profile through a smart array which contains the specific fields, including 'name', 'category', 'species', 'target', 'disease', 'viewer', 'pubchem', 'PDB', 'cas_number', 'SMILES', 'molecular_weight', 'formula', 'alogp', 'hba', 'hbd', 'polar_surface', 'rotatable_bound', 'heavy_atoms', 'rings', 'info' and 'synonyms' (Fig. 7). The user may make a second level filtering pass on the generated results through a keyword search on the smart array in order to separate entries of interest. The HDGUI output from the 'pharmacophore search' differs significantly from the other web tools. It tables the output results in a downloadable smart array with the major fields, such as 'grid', 'SMILES', 'name', 'pubchem', 'pdb', 'ligand-based', 'structural-based' and 'tanimoto80' (Fig. 6). Further characterization of the NPs as drugs in clinical research phases is under way.

An example. The Hippo(crates) interface has been used towards extracting beneficial knowledge and corresponding natural products through various example searches (Species, Target, Disease, Smart, Blast Chem or Pharmacophore) located at http://openscreen.aua.gr/examples.php. For the genus 'Ganoderma', a well-known basidiomycete used in Chinese traditional medicine with pharmacological effects for several ailments. By typing the keyword Ganoderma in the 'Smart Search' tab, and clicking 'Search', the user creates a query for the database. The search of the Hippo(crates) database results, in a list of 281 entries with several natural products related to Ganoderma. Moreover, by inspecting the results and specially the 'Info' list, where information correlated to the query genus is provided, it can clearly be seen that Ganoderma related natural products have therapeutic potential as hepatoprotective, anticancer, anti-inflammatory, immunomodulatory, antioxidant and antiviral agents. Several recent systematic studies have established and confirmed these findings based on the literature $(49,50)$.

\section{Conclusion}

Recent advances in genetics, clinical genomics and personalized medicine have led to the need of discovering effective therapeutic agents for several pathological conditions (51-54). The discovery and correlation of NPs has shed light on the seemingly unrelated correlation between human diseases and certain molecules, leading to novel biologically active drugs. Natural products are considered as a rich source of therapeutic agents endowed with various significant pharmacological properties. The Hippo(crates) database today lists 45,300 NPs and other chemical compounds categorized into 32 major categories. Moreover, several NPs have been associated with various organisms of origin. A total of 22,500 different organisms were associated with various chemical compounds, and more than 32,500 ontologies identified and presented in the 'target' and 'diseases' fields of the database. Currently, several NP databases facilitate the research on the field by classifying the chemical compounds based on 2D and 3D molecular similarity. However, to date, to the best of our knowledge, there was a lack of a NP database that comprises information regarding chemical compounds and pharmacophore models. The present study, with the Hippo(crates) database, aimed to fill this essential gap by providing $>2,500$ representative pharmacophore models. Finally, the HDGUI provides significant assistance for the discovery of NPs as it facilitates access to information relating substances with their known implication in biological activities, originating source, disease association, targeting biomolecule (protein, nucleic acid and carbohydrate), co-crystallized 3D structures, pharmacophoric models, and other beneficial knowledge.

\section{Acknowledgements}

Not applicable.

\section{Funding}

The present study was funded by the project '(OPENSCREEN-GR) An Open-Access Research Infrastructure of Chemical Biology and Target-Based Screening Technologies for Human and Animal Health, Agriculture and the Environment' (grant no. MIS 5002691), which is implemented under the Action 'Reinforcement of the Research and Innovation Infrastructure', funded by the Operational Program 'Competitiveness, Entrepreneurship and Innovation' (NSRF 2014-2020) and co-financed by Greece and the European Union (European Regional Development Fund). The project OPENSCREEN-GR aims to integrate high-capacity screening platforms throughout Greece which jointly use a rationally selected compound collection and offer to researchers from academic institutions, SME's and industrial organizations open access to its shared resources.

\section{Availability of data and materials}

The Hippo(crates) database is publicly available online at: http://www.openscreen.aua.gr/login.php.

\section{Authors' contributions}

LP and EE participated in the construction of the database. LP, AA, EC, KB, DV, TT and EE were involved in the validation and visualization of the database. TT and EE participated in methodology and TT in the Tanimoto analysis. AA, EC, KB and DV searched the literature and performed data collec- 
tion and curation. LP and EE wrote the original draft of the manuscript and were involved in further writing, reviewing and editing along with AA and TT. EE was involved in the conceptualization and design of the study, as well as in funding acquisition. LP and EE have confirmed the authenticity of all the raw data and all authors have read and approved the final manuscript.

\section{Ethics approval and consent to participate}

Not applicable.

\section{Patient consent for publication}

Not applicable.

\section{Competing interests}

The authors declare that they have no competing interests.

\section{References}

1. Hong J: Role of natural product diversity in chemical biology. Curr Opin Chem Biol 15: 350-354, 2011.

2. Bernardini S, Tiezzi A, Laghezza Masci V and Ovidi E: Natural products for human health: An historical overview of the drug discovery approaches. Nat Prod Res 32: 1926-1950, 2018.

3. Beutler JA: Natural Products as a Foundation for Drug Discovery. Curr Protoc Pharmacol 46: 9.11.1-9.11.21, 2009.

4. Newman DJ and Cragg GM: Natural products as sources of new drugs over the last 25 years. J Nat Prod 70: 461-477, 2007.

5. Thomford NE, Senthebane DA, Rowe A, Munro D, Seele P, Maroyi A and Dzobo K: Natural Products for Drug Discovery in the 21st Century: Innovations for Novel Drug Discovery. Int J Mol Sci 19: E1578, 2018.

6. Kayser O, Kiderlen AF and Croft SL: Natural products as antiparasitic drugs. Parasitol Res 90 (Suppl 2): S55-S62, 2003.

7. Salam AM and Quave CL: Opportunities for plant natural products in infection control. Curr Opin Microbiol 45: 189-194, 2018.

8. Sut S, Dall'Acqua S,Zengin G, Senkardes I, Bulut G, Cvetanović A, Stupar A, Mandić A, Picot-Allain C, Dogan A, et al: Influence of different extraction techniques on the chemical profile and biological properties of Anthemis cotula L.: Multifunctional aspects for potential pharmaceutical applications. J Pharm Biomed Anal 173: 75-85, 2019.

9. Salis C, Papageorgiou L, Papakonstantinou E, Hagidimitriou M and Vlachakis D: Olive Oil Polyphenols in Neurodegenerative Pathologies. Adv Exp Med Biol 1195: 77-91, 2020.

10. Treml J, Gazdová M, Šmejkal K, Sudomová M, Kubatka P and Hassan STS: Natural Products-Derived Chemicals: Breaking Barriers to Novel Anti-HSV Drug Development. Viruses 12: E154, 2020.

11. Koumandou VL, Papageorgiou L, Tsaniras SC, Papathanassopoulou A, Hagidimitriou M, Cosmidis N and Vlachakis D: Microbiome Hijacking Towards an Integrative Pest Management Pipeline. Adv Exp Med Biol 1195: 21-32, 2020.

12. Kong DX, Li XJ and Zhang HY: Where is the hope for drug discovery? Let history tell the future. Drug Discov Today 14: $115-119,2009$.

13. Wagner $\mathrm{H}$ and Ulrich-Merzenich G: Synergy research: Approaching a new generation of phytopharmaceuticals Phytomedicine 16: 97-110, 2009.

14. Calixto JB: The role of natural products in modern drug discovery. An Acad Bras Cienc 91 (Suppl 3): e20190105, 2019.

15. Newman DJ and Cragg GM: Natural Products as Sources of New Drugs from 1981 to 2014. J Nat Prod 79: 629-661, 2016.

16. Agrafiotis DK, Bandyopadhyay D, Wegner JK and Vlijmen $\mathrm{H}$ : Recent advances in chemoinformatics. J Chem Inf Model 47: 1279-1293, 2007.

17. Lo YC, Rensi SE, Torng W and Altman RB: Machine learning in chemoinformatics and drug discovery. Drug Discov Today 23: $1538-1546,2018$
18. Muegge I and Mukherjee P: An overview of molecular fingerprint similarity search in virtual screening. Expert Opin Drug Discov 11: 137-148, 2016.

19. Fernandes JPS; The Importance of Medicinal Chemistry Knowledge in the Clinical Pharmacist's Education: The Importance of Medicinal Chemistry Knowledge in the Clinical Pharmacist's Education. Am J Pharm Educ 82: 6083, 2018.

20. Mitsis T, Papageorgiou L, Efthimiadou A, Bacopoulou F, Vlachakis D, Chrousos GP and Eliopoulos E: A comprehensive structural and functional analysis of the ligand binding domain of the nuclear receptor superfamily reveals highly conserved signaling motifs and two distinct canonical forms through evolution. World Acad Sci J 1: 264-274, 2019.

21. Papageorgiou L, Shalzi L, Pierouli K, Papakonstantinou E, Manias S, Dragoumani K, Nicolaides N, Giannakakis A, Bacopoulou F, Chrousos G, et al: An updated evolutionary study of the nuclear receptor protein family. World Acad Sci J 3: 51 , 2021.

22. Chaudhari R, Tan Z, Huang B and Zhang S: Computational polypharmacology: A new paradigm for drug discovery. Expert Opin Drug Discov 12: 279-291, 2017.

23. Pushpakom S, Iorio F, Eyers PA, Escott KJ, Hopper S, Wells A, Doig A, Guilliams T, Latimer J, McNamee C, et al: Drug repurposing: Progress, challenges and recommendations. Nat Rev Drug Discov 18: 41-58, 2019.

24. Vlachakis D, Papageorgiou L, Papadaki A, Georga M, Kossida S and Eliopoulos E: An updated evolutionary study of the Notch family reveals a new ancient origin and novel invariable motifs as potential pharmacological targets. PeerJ 8: e10334, 2020.

25. Awale M, Visini R, Probst D, Arús-Pous J and Reymond JL: Chemical Space: Big Data Challenge for Molecular Diversity. Chimia (Aarau) 71: 661-666, 2017.

26. Voigt JH, Bienfait B, Wang S and Nicklaus MC: Comparison of the NCI open database with seven large chemical structural databases. J Chem Inf Comput Sci 41: 702-712, 2001.

27. Wishart DS, Knox C, Guo AC, Cheng D, Shrivastava S, Tzur D, Gautam B and Hassanali M: DrugBank: A knowledgebase for drugs, drug actions and drug targets. Nucleic Acids Res 36 (Suppl 1): D901-D906, 2008.

28. Wishart DS, Feunang YD, Guo AC, Lo EJ, Marcu A, Grant JR, Sajed T, Johnson D, Li C, Sayeeda Z, et al: DrugBank 5.0: A major update to the DrugBank database for 2018. Nucleic Acids Res 46 (D1): D1074-D1082, 2018.

29. Zeng X, Zhang P, He W, Qin C, Chen S, Tao L, Wang Y, Tan Y, Gao D, Wang B, et al: NPASS: Natural product activity and species source database for natural product research, discovery and tool development. Nucleic Acids Res 46 (D1): D1217-D1222, 2018.

30. Choi H, Cho SY, Pak HJ, Kim Y, Choi JY, Lee YJ, Gong BH, Kang YS, Han T, Choi G, et al: NPCARE: Database of natural products and fractional extracts for cancer regulation. J Cheminform 9: 2, 2017

31. Cheng S, Zhu C, Chu C, Huang T, Kong X and Zhu LC: Prediction of bioactive compound pathways using chemical interaction and structural information. Comb Chem High Throughput Screen 19: 161-169, 2016.

32. Ihlenfeldt WD, Voigt JH, Bienfait B, Oellien F and Nicklaus MC: Enhanced CACTVS browser of the Open NCI Database. J Chem Inf Comput Sci 42: 46-57, 2002.

33. Knox C, Law V, Jewison T, Liu P, Ly S, Frolkis A, Pon A, Banco K, Mak C, Neveu V, et al: DrugBank 3.0: A comprehensive resource for 'omics' research on drugs. Nucleic Acids Res 39 (Database): D1035-D1041, 2011.

34. Hähnke VD, Kim S and Bolton EE: PubChem chemical structure standardization. J Cheminform 10: 36, 2018.

35. Heller SR, McNaught A, Pletnev I, Stein S and Tchekhovskoi D: InChI, the IUPAC International Chemical Identifier. J Cheminform 7: 23, 2015.

36. Reyes-Aldasoro CC: The proportion of cancer-related entries in PubMed has increased considerably; is cancer truly 'The Emperor of All Maladies'? PLoS One 12: e0173671, 2017.

37. Dunn MJ, Jorde LB, Little PFR and Subramaniam S (eds) Encyclopedia of Genetics, Genomics, Proteomics and Bioinformatics, 8 Volume Set. John Wiley \& Sons Ltd., Hoboken, NJ, 2005.

38. Vilar S, Cozza G and Moro S: Medicinal chemistry and the molecular operating environment (MOE): Application of QSAR and molecular docking to drug discovery. Curr Top Med Chem 8: 1555-1572, 2008. 
39. Mhlanga P, Wan Hassan WA, Hamerton I and Howlin BJ: Using combined computational techniques to predict the glass transition temperatures of aromatic polybenzoxazines. PLoS One 8: e53367, 2013.

40. Ihlenfeldt WD, Takahashi Y, Abe H and Sasaki S: Computation and management of chemical properties in CACTVS: An extensible networked approach toward modularity and compatibility. J Chem Inf Comput Sci 34: 109-116, 1994.

41. Huggins DJ, Venkitaraman AR and Spring DR: Rational methods for the selection of diverse screening compounds. ACS Chem Biol 6: 208-217, 2011.

42. Hudson BD, Hyde RM, Rahr E, Wood J and Osman J: Parameter Based Methods for Compound Selection from Chemical Databases. Quant Struct-Act Rel 15: 285-289, 1996.

43. Bajusz D, Rácz A and Héberger K: Why is Tanimoto index an appropriate choice for fingerprint-based similarity calculations? J Cheminform 7: 20, 2015.

44. Wolber G and Langer T: LigandScout: 3-D pharmacophores derived from protein-bound ligands and their use as virtual screening filters. J Chem Inf Model 45: 160-169, 2005.

45. Shaker B, Yu MS, Lee J, Lee Y, Jung C and Na D: User guide for the discovery of potential drugs via protein structure prediction and ligand docking simulation. J Microbiol 58: 235-244, 2020.

46. Zhou Y, Tang S, Chen T and Niu MM; Structure-Based Pharmacophore Modeling, Virtual Screening, Molecular Docking and Biological Evaluation for Identification of Potential Poly: Structure-Based Pharmacophore Modeling, Virtual Screening, Molecular Docking and Biological Evaluation for Identification of Potential Poly (ADP-Ribose) Polymerase-1 (PARP-1) Inhibitors. Molecules 24: E4258, 2019.

47. Bolton EE, Chen J, Kim S, Han L, He S, Shi W, Simonyan V, Sun Y, Thiessen PA, Wang J, et al: PubChem3D: A new resource for scientists. J Cheminform 3: 32, 2011.
48. Kim S, Bolton EE and Bryant SH: Similar compounds versus similar conformers: Complementarity between PubChem 2-D and 3-D neighboring sets. J Cheminform 8: 62, 2016.

49. Wachtel-Galor S, Yuen J, Buswell JA and Benzie IFF: Ganoderma lucidum (Lingzhi or Reishi): A Medicinal Mushroom. In: Herbal Medicine: Biomolecular and Clinical Aspects. Benzie IFF and Wachtel-Galor S (eds). 2nd edition. CRC Press/Taylor \& Francis, Boca Raton, FL, 2011

50. Xu J, Chen F, Wang G, Liu B, Song H and Ma T: The Versatile Functions of G. Lucidum Polysaccharides and G. Lucidum Triterpenes in Cancer Radiotherapy and Chemotherapy. Cancer Manag Res 13: 6507-6516, 2021.

51. Papageorgiou L, Zervou MI, Vlachakis D, Matalliotakis M, Matalliotakis I, Spandidos DA, Goulielmos GN and Eliopoulos E: Demetra Application: An integrated genotype analysis web server for clinical genomics in endometriosis. Int J Mol Med 47: 115,2021

52. Spreafico R, Soriaga LB, Grosse J, Virgin HW and Telenti A: Advances in Genomics for Drug Development. Genes (Basel) 11: E942, 2020.

53. Cardon LR and Harris T: Precision medicine, genomics and drug discovery. Hum Mol Genet 25 (R2): R166-R172, 2016.

54. Sonehara K and Okada Y: Genomics-driven drug discovery based on disease-susceptibility genes. Inflamm Regen 41: 8, 2021.

This work is licensed under a Creative Commons Attribution-NonCommercial-NoDerivatives 4.0 International (CC BY-NC-ND 4.0) License. 